\title{
Cognitive System Framework for Brain-Training Exercise based on Human-Robot Interaction
}

\author{
Antonio Andriella ${ }^{1}$, Carme Torras and Guillem Alenyà
}

Received: date / Accepted: date

\begin{abstract}
Introduction. Every 3 seconds someone develops dementia worldwide. Brain-training exercises, preferably involving also physical activity, have shown their potential to monitor and improve the brain function of people affected by Alzheimer Disease (AD) or Mild Cognitive Impairment (MCI).

Objectives. This paper presents a cognitive robotic system designed to assist mild dementia patients during brain-training sessions of sorting tokens, an exercise inspired by the Syndrom KurzTest neuropsychological test (SKT).

Methods. The system is able to perceive, learn and adapt to the user's behaviour and is composed of two main modules. The adaptive module based on representing the human-robot interaction as a planning problem, that can adapt to the user performance offering different encouragement and recommendation actions using both verbal and gesture communication in order to minimize the time spent to solve the exercise. As safety is a very important issue, the cognitive system is enriched with a safety module that monitors the possibility of physical contact and reacts accordingly.

Results. The cognitive system is presented as well as its embodiment in a real robot. Simulated experiments are performed to i) evaluate the adaptability of
\end{abstract}

This project has received funding from the European Union's Horizon 2020 research and innovation programme under the Marie Sklodowska-Curie grant agreement (No 721619), by the Spanish Ministry of Science and Innovation HuMoUR TIN2017-90086-R, and by the Spanish State Research Agency through the María de Maeztu Seal of Excellence to IRI (MDM-2016-0656).

${ }^{1}$ A. Andriella, C. Torras, G. Alenyà are with Institut de Robòtica i Informàtica Industrial, CSIC-UPC, C/Llorens i Artigas 4-6, 08028 Barcelona, Spain. \{aandriella, torras, galenya\}@iri.upc.edu the system to different patient use-cases and ii) validate the coherence of the proposed safety module. A real experiment in the lab, with able users, is used as preliminary evaluation to validate the overall approach.

Conclusions. Results in laboratory conditions show that the two presented modules effectively provide additional and essential functionalities to the system, although further work is necessary to guarantee robustness and timely response of the robot before testing it with patients.

Keywords Cognitive Robotic System - Cognitive training · HRI - Robot Safety - Socially Assistive Robotics · Adaptive Robot

\section{Introduction}

Assistive Robotics is an emerging area of research due to the rapid growth in the number of elderly people and the demand for more specialized assistance. With the support and assistance of the robot, therapists could provide more effective training and monitor multiple patients simultaneously.

Alzheimer's disease is a degenerative brain disease and the most common cause of dementia. As reported from the World Alzheimer Report 2018 [1], the number of people suffering from Alzheimer's Disease (AD) worldwide is estimated to be around 50 million, more than the population of Spain. This number is projected to increase to more than 132 million by 2050, as populations age. Total direct costs of $\mathrm{AD}$ and dementia are estimated around US $\$ 1$ trillion by 2018 . Dementia is characterized by a decline of memory, language, and other cognitive capabilities that affects a person's ability to perform everyday activities [22]. While there is no cure for these kinds of diseases, non-pharmacological 


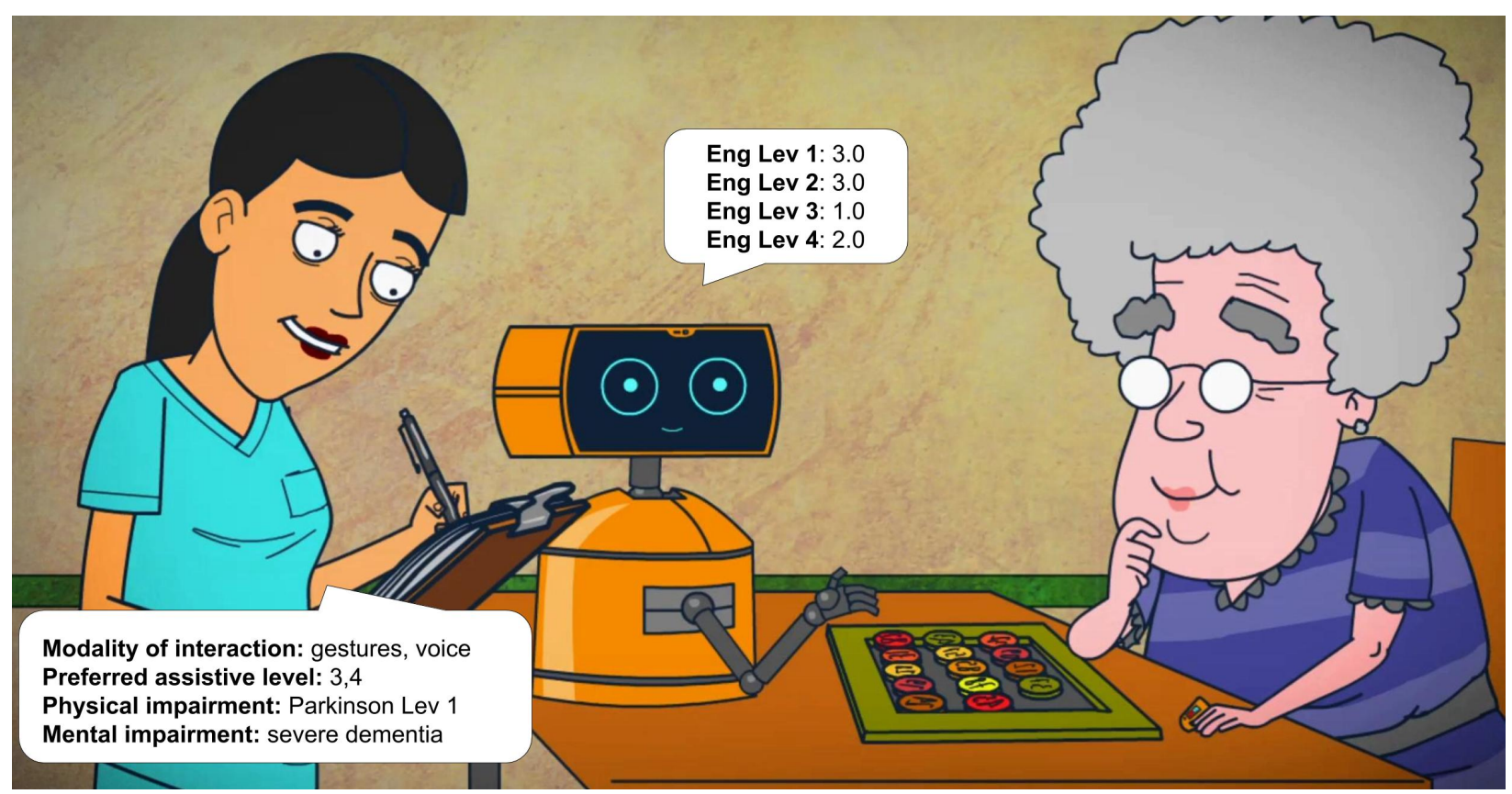

Fig. 1: In the proposed exercise the patient has to place the tokens in the top row of the board in ascending order. The robot observes and provides assistance while the user is playing. The level of assistance (from Lev 1 to Lev 4 ) is selected according to the user performance and moves history. An initial preference on the levels of interaction is provided by the caregiver.

therapies aim to delay the loss of mental abilities, to help patients stay independent in everyday life for as long as possible, and to increase their well-being and quality of life. Non-pharmacological therapy focuses on enhancing mental, physical and emotional activities.

One of the tests that is currently being used to assess the cognitive decline of mild dementia patients is called Syndrom KurzTest (SKT) [18]. SKT is a short neuropsychological test to evaluate cognitive deficits in memory and attention. In this work, inspired by the SKT, we design in collaboration with our partner hospital Fundació $\mathrm{ACE}^{1}$ a brain-training exercise called sorting tokens. In this exercise, the patient has to place a number of tokens in a given order on a board with the help of a cognitive robot.

In order to allow robots to assist humans and cooperate with them in scenarios like rehabilitation, assistive or medical, it is necessary to develop effective and robust methods to provide safety in close-proximity human-robot interaction. In this specific context, humans and robots share the same workspace and may come into contact. We can divide these interactions in three categories: no physical contact, as assumed in this work; contact forces are part of the task; contact forces are part of the guidance or collaboration.

1 http://www.fundacioace.org/en
In this paper, we tackle safety according to the standard ISO 10218 that formalizes the requirements and guidelines for safe design, and to the technical specification ISO/TS 15066 [39] that specifies a collaborative method for "power and force limiting". As a result, we provide a way to execute a motion from the robot that is as accurate and fast as possible while consistent with safety constraints.

We have already presented a general HRI framework [4] to tackle this problem that consists of two different interaction loops: first, the caregiver interacts with the robot to set up the initial desired behaviour of the robot (for example, more helpful or more challenging); second, the robot interacts with the patient while he is playing the sorting tokens exercise.

In this paper we concentrate on the second loop, the interaction between the robot and the patient (see Figure 1), and define a new Cognitive System Framework by extending our previous Human-Robot Interaction (HRI) framework. The two main contributions of this paper are:

i. an adaptive module, which relies on symbolic planning, able to select at each step of the brain-training exercise the most suitable action of engagement to support the patient, 
ii. a safety module that monitors in real-time the user's safety and reacts accordingly adapting the robot behaviour.

The adaptive module will adjust and personalize the robot behaviour based on experienced user interactions, selecting among all the available actions the most appropriate one. The safety component will monitor the user behaviour and react when a hazardous event is detected. It is based on the assumption that the robot is safety-aware. A safety-aware agent knows when its actions, executed in the current state, could hurt or endanger a person and actively refrains from performing them [6].

The Cognitive System is first evaluated in simulation to ensure repeatability when testing its adaptability, and in a simple real robot scenario when validating its feasibility. At this stage, evaluation with real patients is outside the scope of our work. Our aim is not to provide a definitive engineering solution that requires robust perception and robot execution, but rather to present a Cognitive System Framework in which the robot is able to adapt to the user and react to sporadic unexpected behaviours on the basis of previous interaction experiences. With the proposed framework we also aim to provide caregivers with a tool that can be employed for administering brain-training exercises like the one presented here.

\section{Related Work}

Robots are expected to autonomously accomplish a variety of tasks in real world environments that are constantly changing. In order to cope with these requests, robots must not only be provided with predefined rules of behaviour or fixed sets of actions routines, but also they have to be able to perceive, learn and adapt to the surrounding environment. We can define Cognitive Robotics according to Levesque et al. [35] definition: "Cognitive Robotics is the study of the knowledge representation and reasoning problems faced by an autonomous robot in a dynamic and unknown world". Socially Assistive Robots (SAR) in Eldercare aim to endow robots with the ability to support older adults, through social assistance, rather than physical, in convalescence, rehabilitation, and training. However, since in SAR usually robots share the workspace and interact with vulnerable people, they still need to behave safely with respect to them. Moreover, in order to be effective, any kind of therapy provided by the robot has to be tailored to the user's needs.

\subsection{Cognitive Robotic Systems}

A number of cognitive robotic systems have been developed on different robotics platforms, based on logic programming languages (e.g. Situation Calculus, Golog, Prolog or Description Logic).

Carbone et al. [10] describe a model-based approach to flexible behaviours considering the execution context and the goals, and present the main functionalities of a rescue robot system by considering HRI in the domain of the RoboCup Rescue competition. The main advantages of their approach are: i) the system is selfaware about the current situation, at different levels of abstraction; ii) the operator can take advantage of the control system they proposed in order to have a better perception (using e.g. mapping, localization, learning) of the mission status. De Giacomo et al. [14] define a framework for reasoning about actions through a knowledge-base system on a robot with reactive capabilities. The reasoning capability is provided by Propositional Dynamic Logic (PDL). In another work, De Giacomo et al. [15] present a logic framework for representing dynamic systems based on DL, which allows for the formalization of sensing actions. Ferrein et al. [20] propose a novel method of on-line decision theoretic planning and execution on Golog, which is especially appropriate for robotic applications with frequent sensor updates.

Leimagnan et al. [34] present an architecture for the decision layer of social robots. In particular, they focus on the deliberative layer of the robot designed to share space and tasks with a human, and to reason in a way that takes into account human actions and decisions. In the same direction is the work of Devin et al. [16]. They propose a Human-Aware architecture for managing interactions when the robot and the human share the same goal and workspace. To this end, a Human-Aware Task Planner has been used to define the sequence of actions to perform and to decide whether and when the robot should intervene. Bhat et al. [7] present a neural architecture for goal-direct reasoning and cooperation between multiple robots in an industrial task, where two robots work together for assembling objects in a shared workspace.

An interesting work from a different perspective, that uses some concepts from neurobiology of the brain, is proposed by Mohan et al. [42]. In their work they present some preliminary developments on the DARWIN robots in relation to their abilities to learn and reason. The novelty on the proposed approach are: i) the integration in the computational architecture of some ideas of connecomics in order to go beyond the current limitations of the state of the art machine learn- 
ing systems and ii) the incorporation of behavioural studies based on how conceptual knowledge is organized in the brain.

\subsection{SAR for Therapies and Rehabilitation}

The literature on assistive robots for individuals suffering from $\mathrm{AD}$ or $\mathrm{MCI}$ has not been fully investigated in the past years and very little long-term research has been done. Tapus et al. [51] focus on assistive humanmachine interaction methods with the purpose of facilitating research toward SAR systems capable of supporting and assisting people in daily life. Although the application domains are quite different (children with Autism Spectrum Disorders, post-stroke rehabilitation, elderly people with MCI or AD) there is an underlying common need for a system capable of providing several degrees of assistance, such as encouragement and feedback, toward the assigned task or program. Salichs et al. [46] propose a social robot called Mini that is able to administer one-to-one cognitive stimulation therapies to older adults previously defined by caregivers. The robot interacts with the user through different interaction modalities, such as screen, gestures and speech, among all. In [44], Prula and collegues, empower the robot Mini with a bioinspired decision-making system to adapt the robot's behaviour to different user's capabilities with the aim to improve the overall user's experience. Fan et al. [19] develop a robotic system architecture with the purpose of maintaining functional abilities as well as socialization in order adults and achieving long-term engagement. The system defines a multi-user engagement-based mathematical models for robot interaction. The validation of their system shows that robot is positively accepted by older adults with and without cognitive impairment and it can be used for one-to-one and multi-user HRI. Tapus et al. [50] focus on the study of the interactive and cognitive aspects of robot behaviour in an assistive scenario designed for people suffering from $\mathrm{MD}$ and/or $\mathrm{AD}$. The robot acts as music therapist and tries to stimulate the patient through active listening. The objectives are recall, memory and social interaction. McColl et al. [41] present an assistive robot able of providing cognitive assistance, through engagement and motivation, in order to investigate user compliance during meal-time interactions. Martin et al. [38] describe the use of a humanoid robot as a cognitive stimulation tool in therapy of people with MCI. They develop four types of robotherapy sessions: physiotherapy, music, storytelling and logic-language sessions. The preliminary results with patients with moderate dementia show that their neuropsychiatric symptoms tend to improve over those fol- lowing classic therapy methods. A novel approach is presented by Gnjatovic [23]. Here, he introduces a platform that enables the caregiver to design a robot's dialog behaviour. The presented platform is therapistcentered and domain-independent. It enables the therapist to dynamically model the interaction domain and the dictionary, the interaction context, and the different robot's dialogue strategy.

\subsection{Safety in HRI}

The task of maintaining safety in HRI is multidisciplinary in nature, and researchers have approached it in a variety of ways. We divide these approaches into three categories, following the work of Lasota et al. [32].

\subsubsection{Safety Through Control}

The first category is safety through control. In that category, the pre- and post- collision control methods are investigated. This involves methods that limit parameters, such as speed and related force of the robot, or prevent collisions by defining safety regions or guiding the robot away from humans. Broquere et al. [8] introduce a motion trajectory planner to try to satisfy safety and comfort by limiting acceleration and velocity in cartesian space. Laffranchi et al. [30] present an energy-based control strategy to be used in systems in which the robot works very close to or cooperating with humans. Instead of planning trajectories, they propose a method that limits the dangerous behaviour of the robot when there is an impact, by bounding the energy stored into the system to a maximum value. Heinzmann et al. [26] bound the torque commands of a position control algorithm to values that guarantee safety. These restrictions limit the potential impact force generated in the case of a collision with a person. Haddadin et al. [25] present a lightweight robot designed for interactive and cooperative tasks and they show how reactive control strategies could have a significant effect to guarantee safety to the human during the interaction. Lasota et al. [33] present a real-time safety system capable to allow safe humanrobot interaction that works for very low distances of separation between the two bodies, without the need of robot hardware modification or replacement. Golz et al. [24] devise a method to combine collision monitoring and contact estimation from proprioceptive sensation in order to develop a classification system to discriminate between intended and unintended contact types. 


\subsubsection{Safety Through Motion Planning}

The second category of methods is safety through motion planning, in which safer planning is performed in order to avoid possible collision. Sisbot et al. [48] develop a framework for not only safe but also socially acceptable robot motions. To accomplish this, they consider the human kinematics, vision field, posture and the legibility of the robot's actions. Another framework developed by Sisbot et al. [49] combines various aspects of the previous work, and incorporates considerations for making motion more comfortable by limiting jerk and acceleration. Mainprice et al. [37] propose a planner to generate collision free paths that are admisible and understandable to the human. They use constraints like human vision field and separation distance to drive cost-based search in order to plan safe robot motion within cluttered environments. Cambon et al. [9] aim to create a task planner that is aware of the geometrical constraints and the consequences of its actions in the environment. They try to investigate the bond between task planning and manipulation planning that allows for a more powerful treatment of geometric preconditions and effects of robot actions in realistic environments.

\subsubsection{Safety Using Prediction}

The third category is safety using prediction, which involves the prediction of human actions and motions. This method is particularly efficient when humans and robots are working in close proximity, since it is very important to forsee the actions and movements of humans to achieve safety in a dynamic HRI environment. Dominey et al. [17] develop a method for reasoning about the actions performed, which incorporates an interaction history to facilitate anticipatory robot behaviour. Hoffman et al. [27] develop a framework that utilizes a cost-based Markov process to anticipate human actions and select actions based on the robot's confidence in the validity of the prediction and risk. Another method of encoding a human-robot collaborative task with a probabilistic framework is explored by Nikolaidis et al. [43]. They build on a prior investigation, a human-inspired technique that evaluates the convergence of a robot computation teaming model and a human teammate's mental model. Whereas the previous works focus on short-term prediction of actions, $\mathrm{Li}$ and $\mathrm{Fu}$ [36] develop a framework for prediction of longer duration actions by discovering three key aspects of activity: causality, context-cue, and predictability. They propose a method in which the observed action units are used as a context to predict the next possible ac-

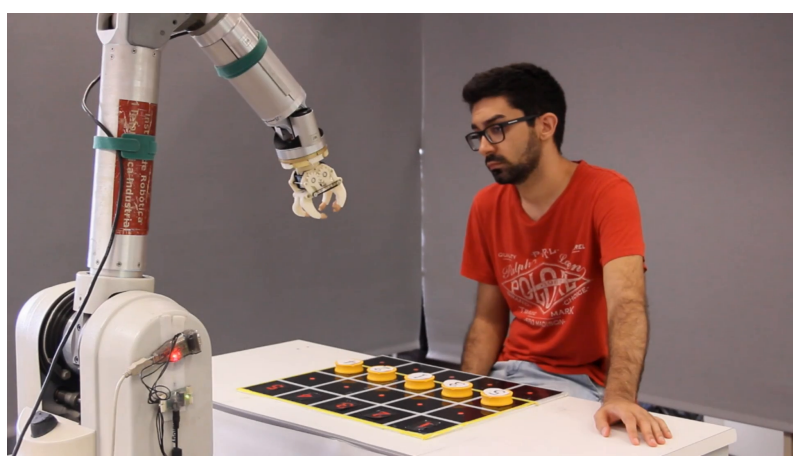

Fig. 2: A Wam $\operatorname{arm}^{2}$ is providing assistance to a user combining speech and gestures based on his performance while he is playing a sorting tokens exercise.

tion unit, or predict the intention of the whole activity. Alami et al. [3] focus their work on the organization of the robot decisional abilities and, in particular, on the management of human interaction as part of the robot control architecture. This architecture allows the robot to accomplish its tasks and also produces behaviours that support its engagement during the interaction with the human. Ragaglia et al. [45] present a methodology to evaluate the severity of an impact between a human worker and an industrial robot. On the basis of this severity evaluation, the robot executes a safety-oriented strategy, ranging from speed reduction, protective stop and trajectory variation. Kulic et al. [29] propose planning and control strategies relying on measures of danger during interaction. The level of danger is estimated based on factors influencing the impact force during a collision, such as the velocity, the distance between the robot and the humans, and the robot inertia.

\section{Cognitive System Framework}

Our Cognitive System is embodied in a robot, which is able to perceive, learn, adapt and react to user behaviours and the status of the exercise. During the exercise, and based on the patient's actions, the robot could assist and support the user combining gestures and verbal interaction modalities. An example of sorting tokens scenario set-up is shown in Figure 2. More details about the sorting tokens exercise will be provided in Section 4.

In order for the Cognitive System to perceive and monitor in real time the state of the board, we endow it with a perception system based on a Kinect camera. The different tokens are detected using classical image processing techniques. We first detect the tokens

\footnotetext{
2 https://www.barrett.com/wam-arm/
} 
shape using Hough Transform, then the number, using an Adaptive Template-Matching algorithm and finally if we use colored tokens we check the result of the classification with color segmentation based on the value of Peak Signal-to-Noise ratio (PSNR) and Structural Similarity (SSIM).

As mentioned in Section 1, the Cognitive System includes two main components: an adaptive and a safety module. The adaptive module is responsible to select at each step of the exercise the most suitable level of engagement for a specific patient (see Section 3.1). The safety module defines a coordinated repertoire of safety procedures that are selected and implemented to respond to potential hazards through expected levels of interaction with the user (see Section 3.2).

\subsection{Adaptive Module}

We represent the entire sorting tokens exercise domain in Planning Domain Definition Language (PDDL 2.1). With this formalism, it is possible to model a highlevel symbolic planning problem and separate it into two major parts: domain description and related problem description.

In our previous framework [4], we already demonstrated that the HRI problem can be effectively modeled using PDDL, and that an off-the-shelf planner can be used then to manage the interaction between the robot and the patient. However, in this framework, the robot can provide assistance only based on the levels set up by the caregiver and it can switch from one level to the other (more assistive) only for wrong moves of the user. In addition, it does not take into account the state of the user or the past interactions.

The extension we propose here is a step forward in this direction: the adaptive module provides reasoning capabilities to the Cognitive System, and is able to take into account the user actions history and the status of the exercise. We assume that the logic of the sorting tokens exercise, that is the correct sequence of moves, is available. This is not a limitation as it can be hardcoded for simple exercises or, in general, obtained using a game solver. Given that, the system knows the next correct move, that we call subGoal (for example, move the token 2 to location 3), the Cognitive System we present here is in charge of deciding the robot-user interactions that help the patient in doing this expected move.

The different actions of engagement we consider are described in Table 1. Four incremental levels of interaction are defined (from the least to the most assistive): i. Encouragement. The robot tries to persuade the user using verbal interactions.

ii. Suggest a subset. The robot suggests a subset of solutions, using speech and gesture it points to an area of the board.

iii. Suggest a solution. The robot communicates verbally the solution to the user and also points to the correct location of the token.

iv. Fully assistive. The robot picks up the correct token and offers it to the user so he has only to place it in the correct location.

The selection of the correct level of engagement has a key importance. Increasing the level could result in a loss of engagement by the patient since the task will be performed almost entirely by the robot. On the other hand, the selection of a lower level of interaction, may result in insufficient assistance by the robot. This could mean the patient feeling frustration for not having achieved the goal, or discouragement for having spent too much time to complete it.

Once the user has performed a move, if it is a correct one, the robot will congratulate and engage him again for the next move. Otherwise, the robot will tell the user the problem and move back the token onto the original location to restart. When the game is completed the robot greets the user giving him information about completion time and number of mistakes.

Algorithm 1 defines the logics of the adaptive system in order to select at each step of the brain-training exercise the best action of engagement given a subGoal $s g$ (defined as the state where one token is replaced in the correct position on the board), a planningDomain, a cost vector $A$ (that contains the value of each action of engagement) and the current state $s$.

Since the problem of finding the best suited action of engagement is defined as an optimization problem, at each step $t$ of the exercise, the planner finds the path with the minimum cost that consists of selecting the action of engagement $e$ with the lower cost and the waiting action for user move $w$ (line 2). After the execution (line 3 and 4), depending on the correctness of the action performed by the user, the cost for that engagement action is updated to learn its effectiveness and adequacy.

The cost $A^{\prime}(e)$ of performing the action of engagement $e$ is defined as

$$
A^{\prime}(e) \leftarrow A(e)+\alpha \cdot[C(e)+\gamma \cdot R(e)-A(e)]
$$

where $A(e)$ is the cost at step $(\mathrm{t}-1)$.

$C(e)$ is defined as:

$C(e)=E(e)+M($ user $)+M($ robot $)$, 


\begin{tabular}{|c|c|c|c|}
\hline Verbal/Gesture & Engagement Level & Robot Interaction & Example of behaviour \\
\hline Verbal & & Instruction & $\begin{array}{l}\text { Hi, I'm Socrates. I will play the exercise with you. } \\
\text { The goal is to place all the tokens in ascending order. } \\
\text { Please try to be as fast as possible. Let's start! }\end{array}$ \\
\hline Verbal & Lev 1 & Encouragement & $\begin{array}{l}\text { Hey try to move a token on the board. } \\
\text { I know you can do it! }\end{array}$ \\
\hline Verbal & Lev 1 & Encouragement & Remember all the tokens must be sorted in ascending order \\
\hline Verbal & Lev 1 & Encouragement & $\begin{array}{l}\text { Learn from your mistakes ... } \\
\text { Try to move the correct one! }\end{array}$ \\
\hline Both & Lev 2 & Suggest subset & $\begin{array}{l}\text { Hey, the solution could be one of these: } \\
\text { Px, Py, Pz. Try to move the token there! }\end{array}$ \\
\hline Both & Lev 2 & Suggest subset & $\begin{array}{l}\text { Hey, try to follow my hand. The solution is between Px and Pz. } \\
\text { Now try to move the token there! }\end{array}$ \\
\hline Both & Lev 2 & Suggest subset & $\begin{array}{l}\text { Hey, keep into account your mistakes ... } \\
\text { Try one of those: Px, Py, Pz. }\end{array}$ \\
\hline Both & Lev 3 & Suggest solution & The correct location for token $\mathrm{Px}$ is $\mathrm{Lx}$. \\
\hline Both & Lev 4 & Fully assistive & This is the correct token, move it in location Lx. \\
\hline Verbal & & Correct move & Congratulations, you have made a successful move. \\
\hline Both & & Wrong move & $\begin{array}{l}\text { Unfortunately, you made the wrong move. } \\
\text { I will move the token back to its initial location. }\end{array}$ \\
\hline
\end{tabular}

Table 1: Example of Robot interaction actions.

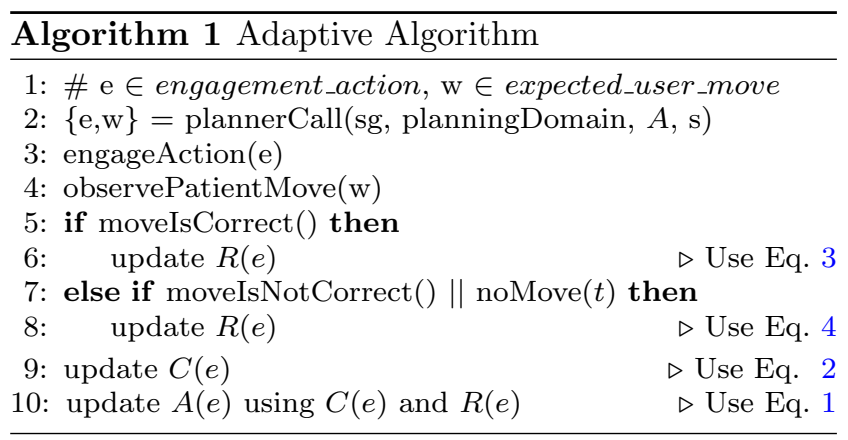

and represents the total time related to an action of engagement $e$ (line 9).

This value is obtained summing up: the time required for an engagement action to be performed $E(e)$, that is variable and depends for example on the modalities of interaction reported in Table 1 (only speech like Lev 1 or speech and gestures like Lev 2, Lev 3 and Lev 4), the reaction time of the user to move the token $M$ (user), that is also used in the report to the caregiver to analyze the user performance, and the action time to move back the token in the original position by the robot if a token has been moved to a wrong location by the user $M$ (robot). This last variable is actually almost a constant and can be set to a defined value at the beginning of the game.
An important part of the adaptive module is the function $R(e)$ which is the update of the reward of the action of engagement $e$ after the user makes a move. That value will be treated as a positive reward if the user makes a successful move (line 6):

$$
R(e)=-\left[\left(n \_b l o c k s \_l e f t / \text { attempts }\right)\right] \cdot(1-G(e))
$$

or on the contrary, as a penalty, if the user makes a wrong move (line 8):

$$
R(e)=\left[\left(1+\left(n \_b l o c k s \_d o n e\right)\right) \cdot(\text { attempts })\right] \cdot G(e)
$$

where $G(e) \in[0,1]$ is a weight relative to an action of engagement, attempts is the number of attempts for placing the correct token on the board, n_blocks_left is the number of tokens that still have to be moved in the correct position on the board and n_blocks_done is the number of tokens already placed in the correct position on the board. The higher the level, the higher should be $G(e)$. Note that difficulty of the exercise changes over time: at the beginning there are more tokens to move so it is harder for the user to guess the correct move, whereas at the last only move a single token is available. 
Also, the Cognitive System should consider differently a failure after a simple engagement action (Lev 1 in Table 1) or after a more helpful engagement (Lev 3/4 in Table 1). $G(e)$ is a way to account for the amount of assistance the user receives.

For example, suppose the robot engages the user providing him a suggestion (Lev 2 Table 1) if the user makes a correct move, $R(e)$ is equal to: $-(5 / 1)^{*}(1-0.5)$ $=-2.5$, where $n \_b l o c k \_l e f t$ is 5 , attempts is 1 and $G(e)$ is 0.5. Suppose now we are at the very end of the game and the user makes a correct move after an engagement action $e$ (Lev 2 Table 1 ), the reward now will be $-(1 / 1) *(1-0.5)=-0.5$. So the lower the level of engagement and the number of attempts and the higher is the number of tokens to place on the board the higher will be the reward.

On the contrary, suppose the robot engages the user providing him a suggestion (Lev 2 Table 1 ) but this time he makes a wrong move, $R(e)$ is equal to: $\left(1^{*} 1\right)^{*}(0.5)$ $=0.5$, where $n_{-}$block_done is 0 , attempts is 1 and $G(e)$ is 0.5. Hypothesize now we are at the very end of the game and the user makes a wrong move after an engagement action $e$ (Lev 2 Table 1$)$, the penalty now will be $\left(5^{*} 1\right) *(0.5)=2.5$. So the higher the level of engagement, the number of attempts and the number of tokens placed on the board, the higher the penalty will be.

Note that we have not interpreted yet the significance of the parameters $\alpha$ and $\gamma$ in Equation 1. It is done now that we have described the rest of the components. The $\gamma$ value defines how much influence is assigned to the estimation of future action for a defined level $e$. A factor of 0 will make the Cognitive System evaluate the cost of the function mainly based on $C(e)$ that is the total time related to an action of engagement e, while a value of 1 will make the system considering the overcome of the previous actions to estimate the next action for level $e$. The $\alpha$ value can be considered a learning factor and determines to what extent the newly acquired information will override the previous one. A factor close to 0 will make the Cognitive System not use any information about the outcome of the previous actions of engagement $e$, while a value close to 1 will make the Cognitive System give more importance to the most recent action.

\subsection{Safety module}

In order to enable a robot to exhibit competent behaviour, with the aim to avoid unwanted physical interactions with humans, we introduce a Safety Module. The presented module has been designed as generic as possible in order to be potentially extended to any kind of users. However, it is worth highlighting that some behavioural characteristics, specifically for patients with dementia, have not been integrated yet. For instance, frustration, confusion, anger, overbalancing, as well as other risks due to the complex clinical needs of the patients.

This module has a monitoring loop to ensure that the dangerous behaviours of the user can be detected. They can be detected at two different times:

- At planning time: the safety problem is detected before starting the engagement action. The Cognitive System will try to persuade the user, or alternatively, change the safety of its own actions as reported in Table 2.

- Execution time: the safety problem is detected while the robot action is being executed. The robot has to possibly stop immediately the execution and react to the dangerous event. We consider the following robot actions: warn the user about his behaviour, move to a safer position, change velocity and acceleration of the trajectory in order to be safer in case of collision, or stop.

The robot should ensure safety compliance in its actions and motion. To this end, a compliant feed-forward controller [11] is used to guarantee a safe contact in case it happens. It works as follows: an inverse dynamic model of the robot had been learned and implemented in order to exert the minimum necessary torque to follow the desired motion. Knowing the dynamics of the robot allows to have a small PD gain term to compensate for model errors and deviations, even detecting contact if necessary as reported by Colome et al. in [12]. This control scheme results in a safe behaviour with low stiffness control, while keeping a good positioning precision. Moreover, the controller can then slow down or even stop motion and switch to gravity compensation mode if there is a detected contact and/or a large deviation from the desired position.

Figure 3 shows the diagram of the safety module. The safety module integrated in our Cognitive System monitors the patient behaviour. When an anomalous behaviour of the user is detected, the robot assigns it a level of safety.

We define a safety value $S F(t, a)$ used to evaluate the next action of the robot, when an unsafe behaviour is detected. This value is defined as

$$
\begin{array}{r}
S F(t, a)=S F(t-1, a)+\theta \\
{\left[S F(t-1, a) \cdot\left(t_{-} \text {elapsed }+S F \_l e v e l\right)\right]}
\end{array}
$$

where $S F(t-1, a)$ is the value of safety at time $t-1$, $S F \_$level $\in\{0,1\}$ and can have different values based 


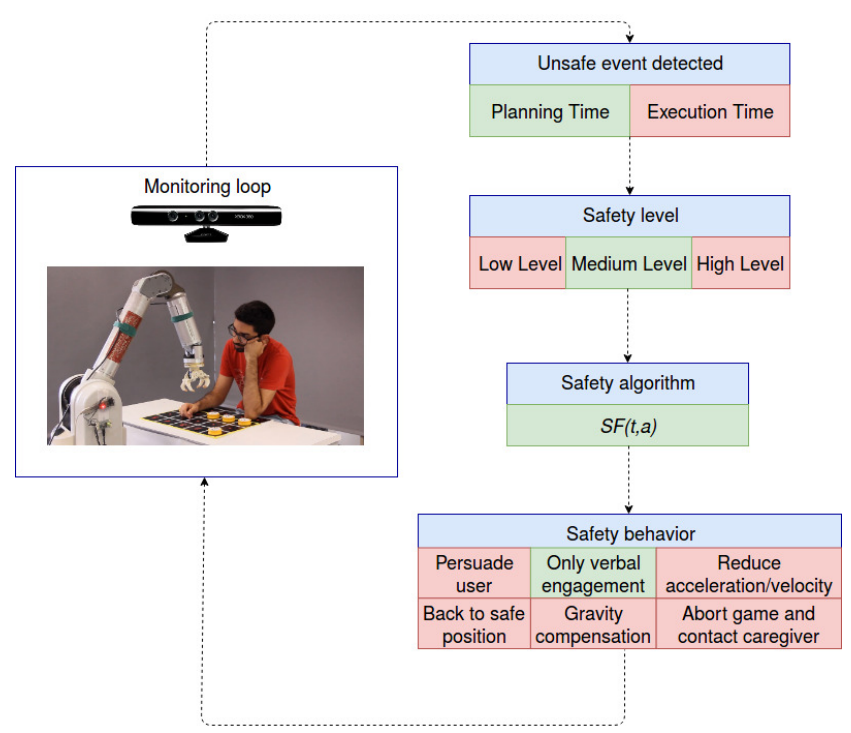

Fig. 3: Macro blocks of the safety module. Highlighted in green the selected events triggered after an hazardous action has been detected: as soon as a dangerous event is detected (Planning Time), based on its risk level (Medium level), the safety algorithm computes the value $S F$ and based on that, the system will determine the safety action to perform (e.g. "Your behaviour is too risky, from now on I won't support you anymore with gestures").

on the corresponding safety level detected (see Figure 4); and t_elapsed is the time the user spends in the unsafe zone. $\theta$ determines how fast to switch from one action to another. In other words, with that parameter we can define the level of severity of the robot. If the computed value is low, the robot will try all the possible safety actions before stopping the exercise and contacting the caregiver. On the contrary, if the value of $\theta$ is high, then the robot will exhibit a more conservative and protective behaviour and will react to the patient's behaviour with safer actions (e.g. no actions of persuading the user, and after the first unsafe event turn the robot to not use anymore gesture, or back to a safe position). We will evaluate the effect of different values of $\theta$ in Section 5 .

The physical meaning of the three safety levels defined is shown in Figure 4. We defined three different levels of safety: low, medium and high. The first level is enabled when the user is too close to the board (Figure 4a). The medium level is activated when the user enters the safety zone and he is on the planned motion trajectory of the robot (Figure 4b). The last level is the most dangerous and is activated when the user is on the robot's motion trajectory and they are so close that they can collide (Figure 4c).

\subsection{Implementation Notes}

The low-level robot movements, as well as the verbal sentences, are programmed using Robot Operating System (ROS). The verbal sentences have been reproduced writing a ROS wrapper for Google Translator. In this way, any language supported by Google Translator can potentially be used to reproduce the robot's voice. A repertory of sentences for each robot action (some of them listed in Table 1) has been created in order to provide the final user with the feeling of interacting with a robot that exhibits more varied behaviours. At the moment, sentences are selected randomly.

The actions that involve gestures (as reported in Table 1 , second column) correspond to engagement levels 2, 3 and 4 . The robot is provided also with an additional action, move token back, that consists to move the token back to its original location when the user's movement is not the correct one. Actions Fully Assistive and move token back require a robot's additional capability, such as grasping with a certain degree of accuracy a token.

Robot motions are generated using Dynamic Movement Primitives (DMP) at a joint level, in which the robot's trajectory is computed with a second order system. In order to have a pure damped attractor to the goal the shaping function is set to 0 [28].

The three different safety levels, defined in the previous section, can be computed automatically based on the distance of the user's hands to the board. We defined a minimum and maximum distance range for each level when the vision system is used and the value is normalized considering the minimum and the maximum distance allowed. One of the most promising alternatives we have evaluated is using OpenPose [47]. OpenPose is a real-time system to detect the human body, including hand and facial key-points on single images. The superposed skeletons on Figure 4 are the representation of OpenPose estimations. As it is possible to note in Figure 4b and Figure 4c, although the performance of OpenPose is really impressive in general scenarios, we faced problems in our setup most of the time due to occlusions of the user's hands (Figure. 4b left hand and Figure $4 \mathrm{c}$ right hand).

At the moment we do not attempt to detect automatically these levels and they are triggered manually by an operator. We are focused on evaluating the reactive behaviour of the robot when an unsafe event is triggered and how the events of unsafe were detected is out of the scope of this paper.

The selection of one of the safety actions listed in Table 2 is based on the value $S F$. In other words, for each safety action in the table we define a range under which that action will be triggered. The switch from 


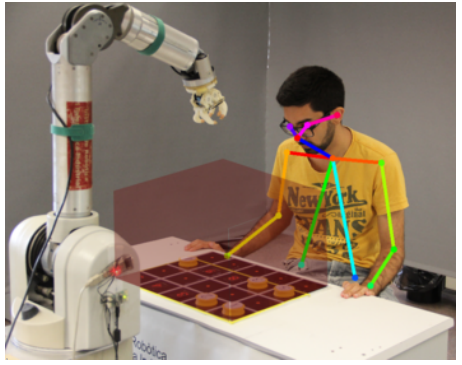

(a) Low level

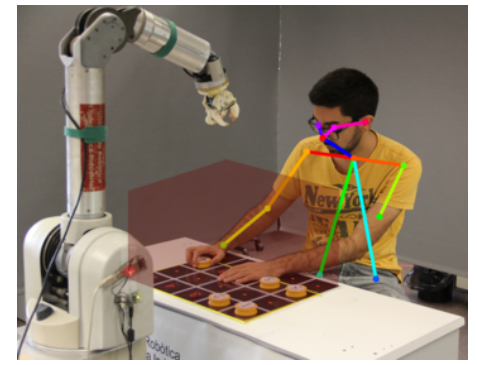

(b) Medium level

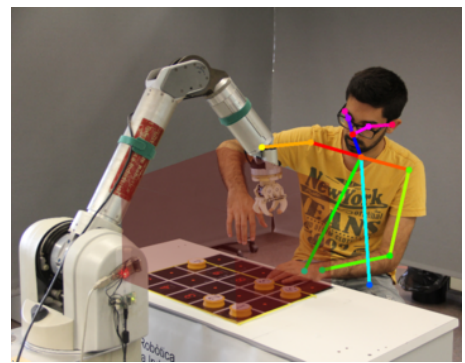

(c) High level

Fig. 4: Three different levels of safety. The virtual red box shows the safety zone, inside which the robot is called to intervene in order to ensure safety for the user. (a) The low level of safety is active since the user hands are too close to the board. (b) The medium level of safety is detected since the user is inside the safety zone but quite far from the planned robot motion trajectory. The robot can not perform any kind of actions until the user does not remove his hands. (c) The user is inside the safety zone and on the robot motion trajectory. The user is so close that they can collide hence the robot stops immediately its motion and reacts taking into account the safety value $S F$ (Equation 5).

\begin{tabular}{ccc}
\hline Safety action & $\begin{array}{c}\text { Unsafe action } \\
\text { timing }\end{array}$ & Safety Preference \\
\hline Persuade user & planning & 1 \\
Only verbal engagement & planning & 2 \\
Abort game and contact caregiver & planning & 3 \\
Persuade user & execution & 1 \\
Reduce acceleration/velocity & execution & 2 \\
Back to a safe position & execution & 3 \\
Gravity compensation & execution & 4 \\
Abort game and contact caregiver & execution & 5 \\
\hline
\end{tabular}

Table 2: List of robot safety actions.

one action to the other is mainly controlled by $\theta$. For example, in the case of unsafe action at planning time, the ranges for each action could be defined as follows:

- 0-0.5 for Persuade user action

- 0.5-0.8 for Only verbal engagement action

- 0.8-1.0 Abort game and contact caregiver action

Moreover, as in the case of the engagement actions (Table 1), the same action can be repeated several times in different ways, in order to provide the user the feeling that the robot is understanding his action and at the same time to gain trustfulness to keep him engaged in the game.

As shown in Table 2, if the returned safety value is very high (meaning the cognitive system has no safety actions to propose), independently of when the hazardous event is detected, as last resort, the robot will abort the game and contact the caregiver, asking for assistance (safety preference 3 for unsafe action detected at planning time and safety preference 5 for unsafe action detected at execution time).

It is important to stress what we already mentioned before (Section 1) about our approach to face risky events. Although path re-planning and find alternative trajectories is a valid strategy, we believe that in our scenario they are not so effective because of the constraints we have in time and space. In time, as the task is to cooperatively complete the game in as little time as possible, it is better to devote the time interacting with the patient than spending time re-planning trajectories. In space, as the working space (the board) is small, the number of different trajectories for grasping a token avoiding the collision is limited and thus usually a valid trajectory cannot be found (space constraint).

Differently our approach is to persuade the user to engage in a better cooperation using symbolic high-level planning to model human-robot interaction. Even when an hazardous event is detected several times, the Cognitive System always attempts to find a different way to convince the user in order to come back to a safe behaviour and continue the game. In addition, it is important to note that both modules cooperate in maintaining the state of the patient and the overall episodic memory. As reported in Table 2, there is one safety action that can affect the robot's adaptive behaviour. Yet, if the safety module decides not to support anymore the user with gestures, then in the next interaction the Cognitive System will provide the user with only verbal assistance. This additional level of safety will affect the robot's interaction modalities and thus the robot won't move anymore its arm either in the case of move token back action, when the user makes a mistake. In that case, the robot tells the user to move the token back to its initial location and it will not provide any further assistance until that action from the user is performed. In all other circumstances in which the robot stops its 
action for a safety reason, the system retries the same assistive action as soon as safe conditions are restored.

\section{The Sorting Tokens Exercise}

The proposed brain-training exercise has been designed for people with $\mathrm{AD}$, and in general for different stages of MCI, in collaboration with Fundació ACE. Fundació $\mathrm{ACE}$ is a research center specialized in the treatment of patients affected by $\mathrm{AD}$ and other dementias, whose therapists, psychologists and neurologists support us providing medical and clinical perspective to our work.

Recent studies suggest that cognitive exercises in the form of games-like can lead to improvement [40], [31], [5] or slow down decline [21] of a number of cognitive functions, such as attention and memory capabilities.

We use SKT as an inspiration for designing the proposed exercise called sorting tokens: short, simple and play-like. SKT is only occasionally administered for assessing patients' cognitive impairment of memory and attention. In contrast, our brain-training exercise can be potentially administered frequently for cognitive training, as well as for evaluation. The exercise has been designed to train patients' cognitive skills (memory and attention) and motor functions (grasping) [13], as well as to evaluate their performance over time.

The objective of sorting tokens is to sort numbered tokens in ascending/descending order on a board, in the shortest time possible while minimising the intervention of the robot. All the tokens have to be sorted by the patient (thus stimulating their cognitive and motor skills) while the robot only provides assistance. To this end, every time the user makes an error, the robot moves the token back to its initial location and provides one of the levels of engagement defined in Table 1 .

It is out of the scope of the current work to design a robotic system able to administer autonomously the full SKT since that would be extremely difficult. On the contrary, we propose an exercise, consisting of a board and tokens, which can be easily modified to have different levels of difficulty and where the robot can be employed by a caregiver to act as an assistant.

It is worth mentioning that although this paper is mainly focused on the loop of interaction between the robot and the patient, in the general approach we rely heavily on the caregiver input (what we call the first loop of interaction) [4]. Thus, the presented work has been designed taking into account that caregivers and patients with dementia agree in receiving assistance from a robot [2]. To this end, we build a robot system from specific caregivers' need: provide more effective cognitive therapies in terms of quality and quantity.
Quality, since we aim to provide the caregiver with interesting output data, like the number of mistakes, reaction time, levels of assistance provided, disengagement occurrence, among others. All these data are impossible to collect during a usual therapist-patient interaction. Quantity, since the robot is able to administer as many exercises as the caregiver sets over time. In this way, the caregiver can potentially set multiple robots and only monitor them while they are administering the exercise to several patients simultaneously.

Hence, we envisage our system as an additional tool to increase the caregiver effectiveness and not as their replacement.

\section{Experiments}

The brain-training scenario used to evaluate the Cognitive System has already been presented in Section 4. Five numbered tokens were arranged randomly on a row on the board. The goal was to place each token in ascending order in the correct location on the board as fast as possible.

As we mainly want to validate the system capabilities, for a deeper and complete analysis we performed most of the experimentation in simulation. The main reasons were efficiency and data analysis. To find the value of the involved parameters and the strategy (sequence of engagement actions) that performed better, it requested several tests and efforts to understand the sensitivity of the parameters. A simulation environment reduced the computational and the execution time and increased the number of possible trials for a more complete evaluation of the results. With the optimal setting, we aimed to validate the effectiveness of our Cognitive System using a real robot manipulator and able users. We recruited 5 participants among students and researchers working in our laboratory with a background in robotics and between the ages of 22 and 40. We asked them to play several times with the system and behave differently in order to evaluate the overall reliability of the entire system.

\subsection{Simulated Experiments}

We performed three different simulated experiments: the first to assess the system response with respect to different user behaviours, the second to evaluate the influence of $\alpha$ and $\gamma$ (see Equation 1) and the last one to tune the $\theta$ (see Equation 5) parameter of the safety module. 


\begin{tabular}{cccc}
\hline Patient Model & Errors & Cumulative Cost & Total Time \\
\hline Very mild dementia & No & 27.45 & $25 \mathrm{sec}$ \\
Mild dementia & Init & 100.45 & $90 \mathrm{sec}$ \\
Mild dementia & End & 230.1 & $105 \mathrm{sec}$ \\
Severe dementia & Many & 406.2 & $145 \mathrm{sec}$ \\
\hline
\end{tabular}

Table 3: Simulation results with different patients model

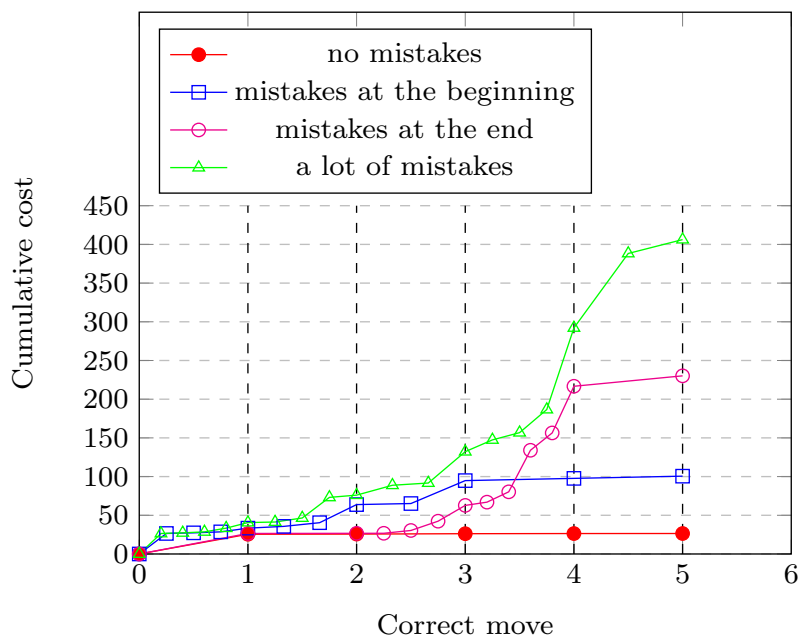

Fig. 5: Analysis of the cumulative cost function for four different patient behaviour (defined in Table 3). Each exercise is composed of 5 different moves. Marks exactly on a number ( 1 to 5 ) correspond to correct moves. Marks between two correct moves correspond to failed user attempts.

\subsubsection{Evaluation of Robot Adaptability}

For the first experiment we defined four user-profiles corresponding to users with different levels of impairment and, consequently, with different capabilities to complete the sorting tokens exercise. We consider 4 different patients (see Table 3): i) patient with very mild dementia who could play almost all the exercise without any additional support except the encouragement, ii) patient with mild dementia that made mistakes in the early stages, iii) patient with mild dementia who made mistakes at the very end, and iv) patient with severe dementia that randomly guessed the moves. To simulate a user profile, a sequence of predefined moves is randomly generated, and defined according to the level of cognitive impairment. Moreover, the variables in the global cost function were initialized and computed as follows: $M$ (robot) was set to 0 for the sake of simplicity; $E(e)$ was set to a constant based on the collected measurements with the robot $(3 \mathrm{~s}, 8 \mathrm{~s}, 10 \mathrm{~s}$ and $15 \mathrm{~s}$ for Lev 1, Lev 2, Lev 3 and Lev 4, respectively) and $M$ (user) was randomly generated.
For a more comprehensive evaluation, it is important to mention that the values of $\alpha=0.8$ and $\gamma=0.6$ have been selected in accordance to the experiments we will propose in Section 5.1.2 in which we evaluated the influence that these two parameters have on the selection of the actions of engagement.

For each user profile, we run 20 exercises and summarize the mean of the numerical results in Figure 5. On the $\mathrm{x}$-axis, we defined the number of correct moves performed from the user, on the y-axis the cumulative cost computed with our adaptive algorithm. The marks on each line define the attempts of each user during the exercise. The number of marks between one correct move to the other defines the number of attempts of the user to guess the correct move. As can be observed comparing the evolution of the four lines, the Cognitive System correctly selected different behaviours for each one of the different patients to provide them with proper assistance in order to complete the exercise. Obviously, this had a considerable impact on the cumulative cost but also on the completion time (Total time in Table 3).

Overall, it can be observed that in the early steps (to reach the correct token 1 and 2) the cumulative cost has not many variations for any of the patients especially for the ones that make a wrong move. The reason is that the cost of performing several engagement actions at this early stage (Equation 3) combined with the penalty of the patient doing wrong moves (Equation 4) is balanced by the difficulty of the brain-training exercise. On the contrary, the value of the cumulative cost has a considerable variation at the end of the exercise (to reach token 4 or 5 ) because failures at the end are penalized as the exercise becomes easier.

The red line (dot mark) shows the plot relative to the user that had very mild dementia and performed the correct move at every step $t$. The line is almost flat, meaning the robot kept the level of engagement to the lowest one and only encouraged the user (Lev 1) to be as fast as possible in solving the game. It is worth to note that even in this situation, a different action of engagement can be performed by the robot. In fact, even though the user makes the correct move, if the user takes too much time to perform a move, at the next step, the robot can decide to furnish him with more assistance in order to speed up the game.

In the second and third use-cases since the simulated patients had mild dementia, their behaviour could be very different based on the mental impairment level. In the second use-case, the user can play the correct moves with confidence only when the exercise became easier, that happened after some tokens were placed on the board with the support of the robot. The blue 
line (square mark) describes the interaction over time of that patient. As can be noted, the user's mistakes did not affect significantly the cumulative cost function since they were performed at the very beginning. The increasing of the curve at the end was mainly due to the changes of engagement levels since the user made few mistakes, thus the cost of performing a more assistive and effective action of engagement had an impact on the cost function.

In the third use-case, we assumed the user could perform the correct moves in the very early stages and then started losing attention. Line magenta (o mark) shows this trend. At the beginning, the robot kept the engagement level at the lower level (Lev 1) since the patient performance in these steps were good enough to decide to switch to a higher level of interaction. After few steps, the patient started making wrong moves, in that case, the robot penalized the user actions, taking into account the number of the tokens left on the board and the number of attempts for each token, as defined in Equation 4. Moreover, at each user mistake, the robot could decide to change its level of interaction toward one more suitable that can unlock the current state of the user but at the cost of performing a higher level of engagement. The sudden rise up of the curve after the user moved the third token in the correct position, shows that behaviour. It is worth highlighting how although the total number of mistakes were almost the same for use-cases 2 and 3, the time to complete the game and the cumulative cost value were considerable different (Table 3). This is mainly because making mistakes at the beginning or at the end of the game had a completely different impact on the cumulative cost (Equation 4).

The last experiment involved a patient with severe dementia that almost randomly guessed the correct move when no assistance was provided by the robot. As it is showed Figure 5 (green line triangle mark), the robot tried to adapt its behaviour to the patient's mistakes after few steps and chose the most suitable level of engagement, according to Equation 1, that might help the user to guess the correct move. The number of attempts as well as the number of mistakes impacted on the cumulative cost, this is the reason why the curve increased especially in the last stages.

\subsubsection{Evaluation of $\alpha$ and $\gamma$}

In the second experiment, we tried to get an intuition about the influence of $\alpha$ and $\gamma$ on the selection of the engagement actions by the robot. As already explained in Section 3.1, $\gamma$ defines to what extent the reward/penalty affects the selection of the next action of assistance.

\begin{tabular}{cccc}
\hline Step & Token & From & To \\
\hline 1 & P2 & L8 & L1 \\
2 & P3 & L7 & L1 \\
3 & P1 & L10 & L1 \\
4 & P2 & L8 & L3 \\
5 & P4 & L6 & L2 \\
6 & P2 & L8 & L2 \\
7 & P4 & L6 & L4 \\
8 & P3 & L10 & L3 \\
9 & P4 & L6 & L5 \\
10 & P4 & L6 & L4 \\
11 & P5 & L9 & L5 \\
\hline
\end{tabular}

(a)

\begin{tabular}{cccc}
\hline Step & Token & From & To \\
\hline 1 & P1 & L10 & L1 \\
2 & P2 & L8 & L2 \\
3 & P4 & L6 & L3 \\
4 & P5 & L9 & L3 \\
5 & P4 & L6 & L3 \\
6 & P3 & L7 & L3 \\
7 & P5 & L6 & L4 \\
8 & P5 & L9 & L4 \\
9 & P5 & L9 & L4 \\
10 & P4 & L6 & L4 \\
11 & P5 & L9 & L5
\end{tabular}

(b)
Table 4: In Tables a and b, we list the sequence of actions performed by two different users. The correct moves are highlighted in gray. In Table a, the user is able to perform the correct moves mainly at the beginning, while, in Table b, the user performed the correct moves mainly at the end of the game.

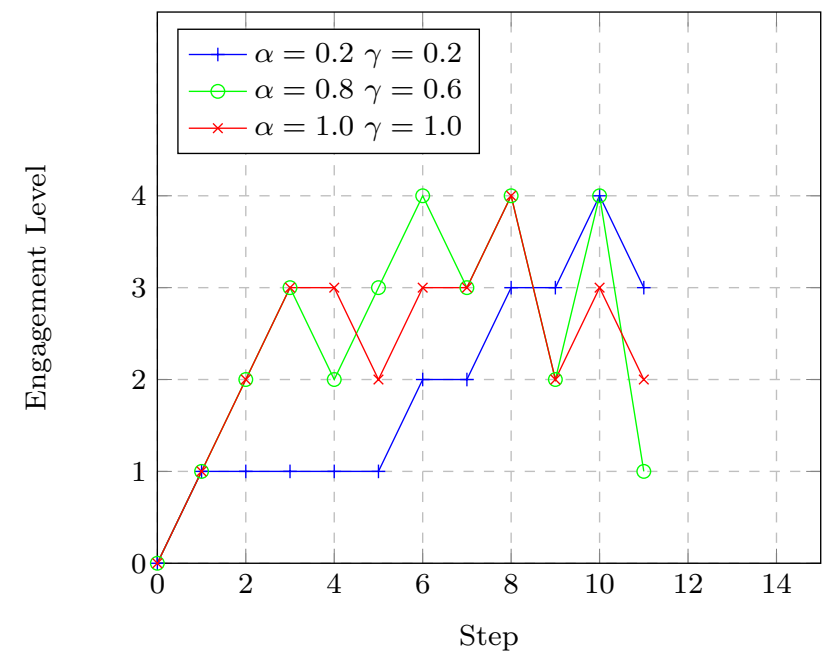

Fig. 6: Analysis of $\alpha$ and $\gamma$ with a patient that makes mistakes at the beginning (the sequences of user moves are reported in Table $4 \mathrm{a}$ ).

While $\alpha$ rules how much influence is given to the new information acquired. We assumed to have two different users as in the previous experiment, the first one that made mistakes at the very beginning of the game and the latter that made mistakes at the very end (as reported in Table 4). The objective was to evaluate in both use-cases the robot actions of engagement when $\alpha$ and $\gamma$ are 0.2, when $\alpha$ and $\gamma$ are 1.0 and when $\alpha$ is 0.8 and $\gamma$ is 0.6 . These last values were selected to be used in all the other experiments since we believe they achieved a reasonable trade-off between two opposite robot behaviours that we will show in the next paragraphs.

In the first case (Table $4 \mathrm{a}$ ) as it is possible to notice in Figure 6, under $\gamma=0.2$ and $\alpha=0.2$ (blue line plus mark) the robot had a conservative strategy, that means 


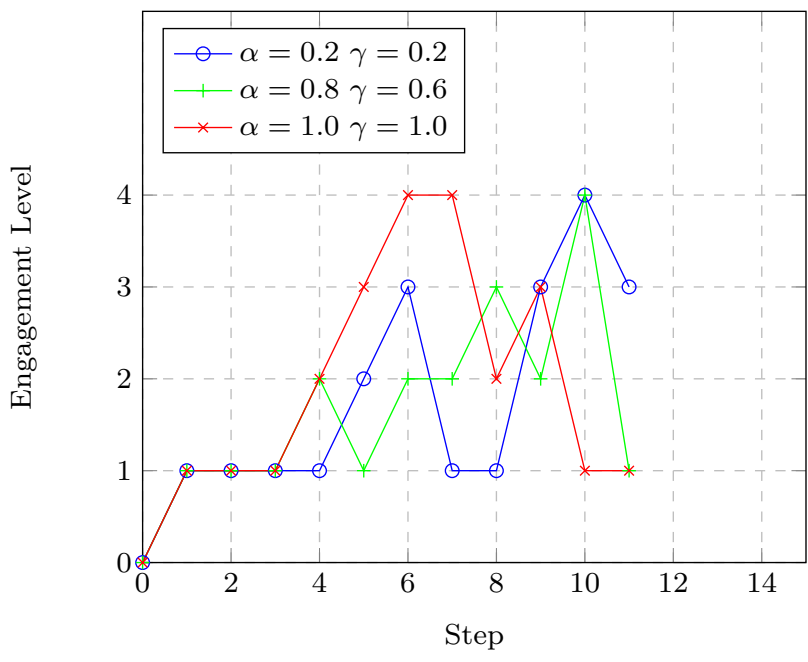

Fig. 7: Analysis of $\alpha$ and $\gamma$ with a patient that makes mistakes at the very end steps (The sequences of user moves are reported in Table $4 \mathrm{~b}$ ).

it needed to fail several times on the same level before switching to the next one. That kind of behaviour is shown in the first three steps, in which, even though the user failed, the robot still tried to re-engage him with the same level of assistance. The reason is evident if we look at Equation 1. Setting low values for $\gamma$ and $\alpha$, caused the Cognitive System to consider very little the information relative to the action outcome (Equations 3 and 4) and the global cost of the action (Equation 2). A completely different behaviour of the robot was obtained with $\gamma=1.0$ and $\alpha=1.0$. The robot was more reactive on each step and, as soon as the user failed, it shifted to the next level of engagement. Another important aspect is the memory of the previous action outcome. For each step in which the user succeeded (he made the correct move), the robot tried to re-engage him with the same level, because it was efficient and rewarded from the algorithm (steps 3-4, 6-7) and only in the last step it decided to provide less support since the game was easier. The main difference with the former case $(\alpha=0.2$ and $\gamma=0.2)$ is that now, the system gave more importance to the outcome of the most recent actions and thus it was able to immediately react to them. As a final test, we evaluated the robot actions of engagement when $\gamma=0.6$ and $\alpha=0.8$. As can be seen in Figure 6 (green line circle mark) the robot behaviour can be considered a balance between the two very different strategies presented in the previous tests. On one hand, the robot tries to provide coherent assistance to the user changing from one level to the other according to his moves. On the other hand, it attempts to challenge him by not providing too much assistance.
In Figure 7 we made the same analysis but with a different patient profile. Here the patient made more mistakes in the final steps as reported in Table $4 \mathrm{~b}$.

In this situation the behaviour of the robot, when $\gamma=0.2$ and $\alpha=0.2$ was a bit different (blue line dot mark). The main reason behind a different behaviour is that now the robot, in contrast to the previous case, made the correct moves in the first steps (step 1, 2). It can be noted that even though it considered very little the previous action outcome, in that specific case, because the first level of engagement resulted in a successful strategy for the first two moves of the user, the robot adopted it as a preferred action even in the next steps $(4,7,8)$. The main reason is straightforward if we look at Equation 3. If the robot action of engagement is successful means the user perform the correct move, thus the system will reward it with a very high value due to the higher complexity of the game at the beginning.

On the contrary, the behaviour of the robot when $\gamma=1.0$ and $\alpha=1.0$ it was as we expected. After the first two successful engagements at step 1 and 2, the robot furnished again the same level (Lev 1) but the user at this step made the wrong move. Hence, at the next step, it decided to provide him with a higher level of assistance (step 4, Lev 2). It is noteworthy that even though at step 3, the robot assistance was not helpful to the user, it still remains the preferred one. This is because Lev 1 was very effective at the very first steps and the adaptive function (Equation 1) tends to promote it with respect to the others. As in the previous case, as soon as the robot found a successful engagement action it maintained it for the next user move (step 6-7). While the user performed a sequence of wrong moves (step 7-8-9-10), the robot tried to switch from one level of engagement to the other, but after several unsuccessful attempts (trying again Lev 3 that was successful at step 6) it decided to move back to the level that had performed better so far; this explains why it came back to engagement level 1 at stage 10 and 11 in the last two moves. As in the previous use-case, we analyzed the robot action of engagement when $\gamma=0.6$ and $\alpha=0.8$. The robot behaviour was, as expected, a trade-off between the two opposite behaviours presented before. Instead of proposing the same action of engagement several times $(\gamma=0.2$ and $\alpha=0.2)$ or engaging the user immediately with a higher level of assistance $(\gamma=1.0$ and $\alpha=1.0)$, its behaviour seemed to be a balance between these two strategies. 


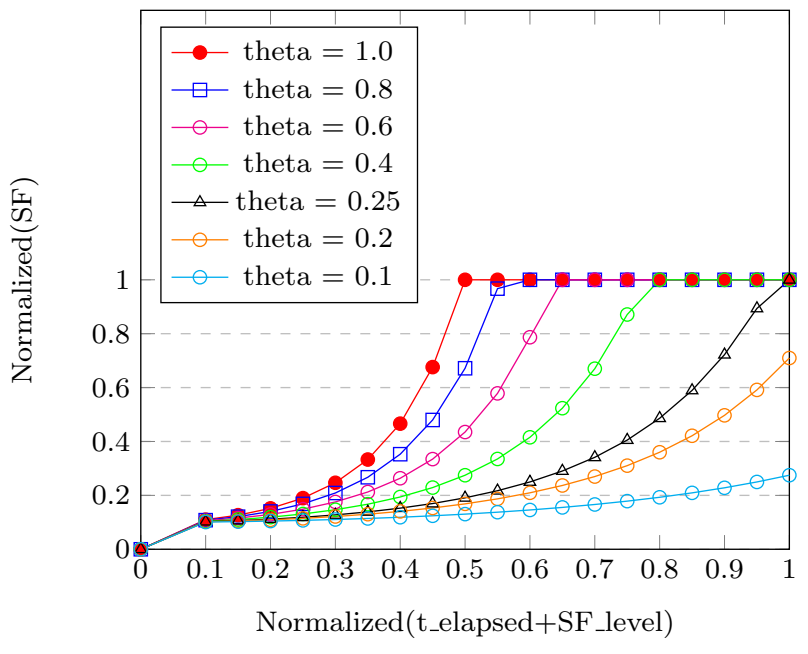

Fig. 8: Analysis of $\theta$ in Equation 5. To each different range of value of $\theta$ corresponds different robot behaviour and consequently different safety actions.

\subsubsection{Evaluation of the $\theta$ Parameter}

In the third experiment, we evaluated the effectiveness of our safety module. The $\theta$ parameter defines the different behaviours of the robot when an unsafe action of the user is detected. In Figure 8 we show how a different value of $\theta$ has a significant influence on the choice of a defined safety action. On the x-axis, we define the normalized value of summing up the elapsed time $t$ plus the level of safety. On the y-axis, the safety value $S F$ is normalized between 0 and 1 . The initial value of $S F$ at time $t=0$ was set to 0.1 .

For completeness, it is worth to mention that each action has a subset of possible sub-actions. For example, with the "persuade user" action, according to the number of times that action is requested, the safety module will produce a different sentence based on the previous failure/s. A sentence could be generated with a hint about the time elapsed as well as the number of tokens left or the user performance at that time.

Another example is the "reduce acceleration/velocity" action. In this case, if the action is called more than one time, the robot will further decrease its arm speed keeping into account the previous failure/s.

It can be observed, from the plots in Figure 8, that for low values of $\theta(<0.25), S F$ is quite small. In this case, the safety module requires several attempts by the robot to switch to a safer action. For instance, for $\theta=0.10$, the robot chose a safety action several times and only at the very end, it moved to safer behaviours, such as moving the arm back to its initial position or in gravity compensation. In spite of this, its behaviour is limited to a fraction of the available actions defined in Table 2. This is because the maximum value of $S F$ is 0.3. Although the strategy to avoid higher levels of safety could help to save time, sometimes it doesn't pay off in terms of persuasiveness and some user's behaviour could not be managed with the adequate safety action. On the other hand, a $\theta$ value greater than 0.4 , signifies a more safety assertive behaviour, in which the robot switches to a safer behaviour very rapidly, only trying to persuade the user few times. For instance, in the case of $\theta$ equal to 0.6 , for values of (t_elapsed $+S F \_$level $)$ greater than 0.65 , corresponds $S F$ equal to 1 (highest safety preference in Table 2). This means that, the safest possible action was performed by the robot, such as to contact the caregiver to ask for assistance. In conclusion, we found that a value of $\theta$ between 0.22 and 0.28 was a good trade-off in terms of robot actions severity. Hence, we used that value in the real experiments.

\subsection{Real Robot Experiment}

The simulations performed in the previous sections can be used to set up the $\alpha, \gamma$ and $\theta$ parameters, as well as the initial cost of performing an action of engagement $A(e)$, if the user profile is given. We envisage that this is a piece of information that the caregiver can provide, as suggested in our previous work [4].

Here we validate our approach using a real robot and show safety-critical situations. Since we administered the test to able users, at this stage we did not include the caregiver in the loop and we set up the initial values of vector $A(e)$ equal to 0 . Moreover, according to the results of the simulated experiments, we set $\alpha, \gamma$ and $\theta$ equal to $0.8,0.6$ and 0.24 , respectively.

At the beginning the robot explained the rules of the exercise to the user, alerting him to not interfere with its actions for safety reasons. Once the exercise is running, the robot starts to interact with the user using the lower level of engagement (Lev 1 as reported in Table 1). As soon as the user makes a wrong move the Cognitive System adapts its behaviour eventually changing to a higher level of interaction (Lev 2, Lev 3 , or Lev 4 ). We provide a video ${ }^{3}$ showing one of the executions where can be seen that the system behaves as expected and adapts to the user.

As practical lessons learned, we use a two-finger gripper to manipulate the tokens that sometimes may fail because the positioning relies on an external camera and slight calibration errors may introduce error.

During the experiment, the safety module is called on purpose several times to evaluate how it adapted

\footnotetext{
3 https://youtu.be/xtS1yDIyrmQ
} 


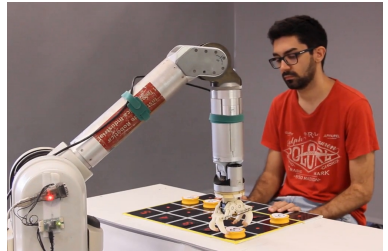

(a) (b)

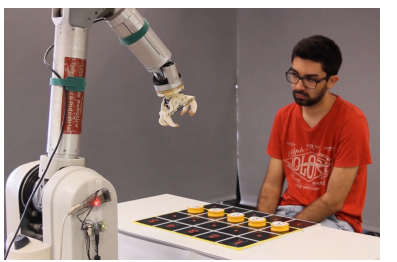

Fig. 9: Example of safety action: back to initial position. (a) The robot detects a wrong move of the user and picks the token to move it back in the original location, but it stops its movement because the user is inside the safety zone. (b) The robot comes back to a safe position and waits until the safety zone is free again.

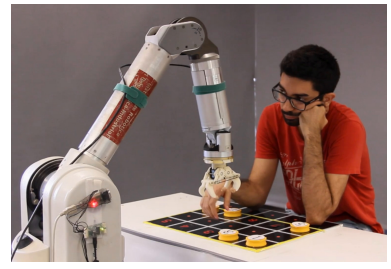

(a)

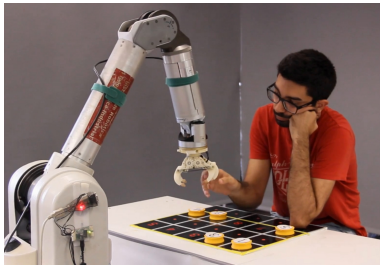

(b)
Fig. 10: Example of safety action: gravity compensation. (a) The robot is suggesting a subset of solutions and pointing them on the board, until the user moves his hands on the board. (b) The robot switches its arm in gravity compensation since the user behaviour was too risky.

in time to show the difference, in term of actions performed, when the event was detected at planning or at execution time.

The robot made several attempts to persuade the user through predefined verbal sentences, with the aim to quickly restore a safe context. When the user behaviour started to be too risky over time, it decided to perform safer actions. In Figure 9, the robot detected a wrong move from the user and tried to move back the token to the original location. While it was performing its movement (Figure 9a), the user moved his hands inside the safety zone. The robot stopped its arm and announced to the user that it moved back to a safety position (Figure 9b). The robot started playing again the exercise, as soon as the safety conditions were restored. During the brain-training exercise it still tried to support and help the user to behave in a safe manner, since with his behaviour, he was putting himself in a potentially dangerous situation as reported in Figure 10a. At that moment the robot put its arm in gravity compensation (Figure 10b), to avoid that any movement per- formed at execution time could cause physical contact with the user. Although our safety system is conceived to avoid any kind of physical contact, it could happen that the user touches accidentally or on purpose the robot arm; in this case, having an action such as gravity compensation should help to prevent injuries.

We provide a video ${ }^{4}$ showing an execution where all safety modes executed by the real robot can be observed.

\section{Conclusions}

In this work, we aim to propose a robotic system as a tool that can be employed by caregivers to administer brain-training exercises to patients affected by different forms of dementia. In the proposed scenario, the user is called to solve the exercise of placing $n$ tokens in ascending order on the board in the shortest possible time with the support and assistance of a robot. We present a new Cognitive System Framework by extending our previous HRI framework [4] adding two components: an adaptive module, that selects the most suitable level of engagement according to the user actions and a safety module that allows the robot to monitor the user performance and react when a hazardous event is detected.

We focus explicitly on representing the key concepts for specifying the adaptive and safety-aware control modules, like the definition of the levels of engagement combining verbal and gesture communication and the safety-relevant events, which are all formalized in symbolic high-level planning language.

In order to dynamically adapt to the behaviour of the user, we have developed two algorithms. The adaptive algorithm, which is able to learn and adapt the robot engagement actions to the user behaviour in order to provide enough assistance to complete the game. The safety algorithm, which constantly monitors the user and selects the most appropriate action to react to an unsafe event.

We showed how the modules can be integrated into our HRI framework, giving additional and essential functionalities to the whole system. Finally, in Section 5 we evaluated our modules on simulation, focusing mainly on the adaptability of the system to different patient use-cases and on validating the coherence of the safety module. Then we performed some experiments in a real scenario with able users to validate the overall approach.

Before the system can be evaluated with real patients, some engineering work has to be done in order to improve the robustness of the perception system

\footnotetext{
4 https://youtu.be/pQ-RM_l1YkI
} 
and the overall time response of the robot, mainly in the speed used to perform some of the trajectories. A further improvement to increase user acceptance and engagement over time will be also explored. A readyto-use prototype would involve a cheaper and more appealing robot embodiment compared to the one used in this work.

Since safety is really important, especially when a robot interacts with patients s, our next goals are: on one hand, to extend the safety module in order to take into account other risks that can derive from the clinical needs of the patients with dementia; and, on the other hand, to evaluate the behaviour of the robot when a physical contact is detected, since sometimes we can't avoid it. The idea is to extend the current safety module with additional functionalities that can manage these other kinds of unsafe events. For instance, using a force sensor we can measure how much effort the user is applying on the gripper and trigger several robot behaviours.

\section{Funding}

This project has been partially funded by the European Union's Horizon 2020 research and innovation programme under the Marie Skodowska-Curie grant agreement SOCRATES (No 721619), by the Spanish Ministry of Science and Innovation HuMoUR TIN2017-90086$\mathrm{R}$, and by the Spanish State Research Agency through the María de Maeztu Seal of Excellence to IRI (MDM2016-0656).

\section{Acknowledgements}

We would like to thank Carla Abdelnour, Joan Hernandez and Natalia Tantinya from Fundació ACE for the fruitful discussions and the help in the design of the sorting tokens exercise.

\section{Compliance with Ethical Standards}

\section{Conflict of interest}

The authors declare that they have no conflict of interest.

\section{Ethical approval}

All participants were able adult students at the Institut de Robòtica i Informàtica Industrial in Barcelona (Spain) and were informed that if at any point they did not wish to continue with the study, they could withdraw from the experiment. The study was approved by the Ethical Committee of the Consejo Superior de Investigaciones Cientficas (reference code 048/2018).

\section{Informed consent}

Informed consent was obtained from all individual participants included in the study.

\section{References}

1. World Alzheimer Report The state of the art of dementia research: New frontiers. Alzheimer's Disease International (ADI) pp. 1-48 (2018). URL https://www.alz. co.uk/research/WorldAlzheimerReport2018.pdf

2. Abdelnour, C., Tantyna, N., Hernandez, J., Giakoumis, D., Ribes, J.C., Gerlowska, J., Skrobas, U., Korchut, A., Grabowska, K., Szklener, S., Hernandez, I., RosendeRoca, M., Mauleon, A., Vargas, L., Alegret, M., Espinosa, A., Ortega, G., Sanchez, D., Rodriguez-Gomez, O., Sanabria, A., Perez, A., Canabate, P., Moreno, M., Preckler, S., Ruiz, A., Rejdak, K., Tzovaras, D., Tarraga, L., Boada, M.: Are there differences in the opinion of patients with Alzheimers Disease and their caregivers about having support from a service robot at home? Alzheimer's \& Dementia: The Journal of the Alzheimer's Association 13(7), P1412-P1413 (2017)

3. Alami, R., Clodic, A., Montreuil, V., Sisbot, E., Chatila, R.: Task planning for human-robot interaction. Proceedings of the 2005 joint conference on Smart objects and ambient intelligence: innovative context-aware services: usages and technologies (october), 8185 (2005)

4. Andriella, A., Alenyà, G., Hernández-Farigola, J., Torras, C.: Deciding the different robot roles for patient cognitive training. International Journal of Human Computer Studies 117, 20-29 (2018)

5. Bahar-Fuchs, A., Webb, S., Bartsch, L., Clare, L., Rebok, G., Cherbuin, N., Anstey, K.J.: Tailored and Adaptive Computerized Cognitive Training in Older Adults at Risk for Dementia: A Randomized Controlled Trial. Journal of Alzheimer's Disease 60(3), 889-911 (2017)

6. Beetz, M., Bartels, G., Albu-Schaffer, A., BalintBenczedi, F., Belder, R., Bebler, D., Haddadin, S., Maldonado, A., Mansfeld, N., Wiedemeyer, T., Weitschat, R., Worch, J.H.: Robotic agents capable of natural and safe physical interaction with human co-workers. In: 
IEEE International Conference on Intelligent Robots and Systems, vol. 2015-Decem, pp. 6528-6535 (2015)

7. Bhat, A.A., Mohan, V.: Goal-Directed Reasoning and Cooperation in Robots in Shared Workspaces: an Internal Simulation Based Neural Framework. Cognitive Computation 10(4), 558-576 (2018)

8. Broquère, X., Sidobre, D., Herrera-Aguilar, I.: Soft Motion Trajectory Planner for Service Manipulator Robot. ArXiv e-prints (2009)

9. Cambon, S., Alami, R., Gravot, F.: A Hybrid Approach to Intricate Motion, Manipulation and Task Planning. The International Journal of Robotics Research 28(1), 104-126 (2009)

10. Carbone, A., Finzi, A., Orlandini, A., Pirri, F., Ugazio, G.: Augmenting situation awareness via model-based control in rescue robots. In: 2005 IEEE/RSJ International Conference on Intelligent Robots and Systems, IROS, pp. 1549-1555. IEEE (2005)

11. Colome, A., Pardo, D., Alenya, G., Torras, C.: External force estimation during compliant robot manipulation. In: Proceedings - IEEE International Conference on Robotics and Automation, pp. 3535-3540. IEEE (2013)

12. Colome, A., Planells, A., Torras, C.: A friction-modelbased framework for Reinforcement Learning of robotic tasks in non-rigid environments. 2015 IEEE International Conference on Robotics and Automation (ICRA) (1), 5649-5654 (2015)

13. De Boer, C., Echlin, H.V., Rogojin, A., Baltaretu, B.R., Sergio, L.E.: Thinking-While-Moving Exercises May Improve Cognition in Elderly with Mild Cognitive Deficits: A Proof-of-Principle Study. Dementia and Geriatric Cognitive Disorders Extra 8(2), 248-258 (2018)

14. De Giacomo, G., Iocchi, L., Nardi, D., Rosati, R.: Moving a robot: The KR\&R approach at work. In: Proceedings of the Fifth International Conference on the Principles of Knowledge Representation and Reasoning (KR'96), pp. 198-209 (1996)

15. De Giacomo, G., Iocchi, L., Nardi, D., Rosati, R.: Planning with Sensing for a Mobile Robot. Proceedings of ECP-97: Fourth European Conference on Planning, pp. 158-170 (1997)

16. Devin, S., Clodic, A., Alami, R.: About Decisions During Human-Robot Shared Plan Achievement: Who Should Act and How? Lecture Notes in Computer Science (including subseries Lecture Notes in Artificial Intelligence and Lecture Notes in Bioinformatics) 10652 LNAI, 453463 (2017)

17. Dominey, P.F., Metta, G., Nori, F., Natale, L.: Anticipation and initiative in human-humanoid interaction. In: 2008 8th IEEE-RAS International Conference on $\mathrm{Hu}-$ manoid Robots, Humanoids 2008, pp. 693-699. IEEE (2008)

18. Erzigkeit, H.: SKT: a short cognitive performance test for assessing deficits of memory and attention. User's Manual. International Psychogeriatrics 9(S1), 115-121 (2001)

19. Fan, J., Bian, D., Zheng, Z., Beuscher, L., Newhouse, P.A., Mion, L.C., Sarkar, N.: A Robotic Coach Architecture for Elder Care (ROCARE) Based on Multi-user
Engagement Models. IEEE Transactions on Neural Systems and Rehabilitation Engineering PP(99) (2016)

20. Ferrein, A., Fritz, C., Lakemeyer, G.: On-line DecisionTheoretic Golog for Unpredictable Domains. KI2004 Advances in Artificial Intelligence 3238, 322-336 (2004)

21. Gates, N., Valenzuela, M.: Cognitive exercise and its role in cognitive function in older adults. Current Psychiatry Reports 12(1), 20-27 (2010)

22. Gaugler, J., James, B., Johnson, T., Scholz, K., Weuve, J.: 2016 Alzheimer's disease facts and figures. Alzheimer's and Dementia 12(4), 459-509 (2016)

23. Gnjatović, M.: Therapist-Centered Design of a Robots Dialogue Behavior. Cognitive Computation 6(4), 775788 (2014)

24. Golz, S., Osendorfer, C., Haddadin, S.: Using tactile sensation for learning contact knowledge: Discriminate collision from physical interaction. 2015 IEEE International Conference on Robotics and Automation (ICRA) pp. 3788-3794 (2015)

25. Haddadin, S., Albu-Schäffer, A., De Luca, A., Hirzinger, G.: Collision detection and reaction: A contribution to safe physical human-robot interaction. In: 2008 IEEE/RSJ International Conference on Intelligent Robots and Systems, IROS, pp. 3356-3363. IEEE (2008)

26. Heinzmann, J., Zelinsky, A.: Quantitative Safety Guarantees for Physical Human-Robot Interaction. The International Journal of Robotics Research 22(7-8), 479-504 (2003)

27. Hoffman, G., Breazeal, C.: Cost-Based Anticipatory Action Selection for HumanRobot Fluency. IEEE Transactions on Robotics 23(5), 952-961 (2007)

28. Husain, F., Colome, A., Dellen, B., Alenya, G., Torras, C.: Realtime tracking and grasping of a moving object from range video. Proceedings - IEEE International Conference on Robotics and Automation pp. 2617-2622 (2014)

29. Kulić, D., Croft, E.: Pre-collision safety strategies for human-robot interaction. Autonomous Robots 22(2), 149-164 (2007)

30. Laffranchi, M., Tsagarakis, N.G., Caldwell, D.G.: Safe human robot interaction via energy regulation control. Intelligent Robots and Systems, 2009. IROS 2009. IEEE/RSJ International Conference on pp. 35-41 (2009)

31. Lampit, A., Valenzuela, M., Gates, N.J.: Computerized Cognitive Training Is Beneficial for Older Adults. Journal of the American Geriatrics Society 63(12), 2610-2612 (2015)

32. Lasota, P.A., Fong, T., Shah, J.A.: A Survey of Methods for Safe Human-Robot Interaction. Foundations and Trends in Robotics 5(3), 261-349 (2017)

33. Lasota, P.A., Rossano, G.F., Shah, J.A.: Toward safe close-proximity human-robot interaction with standard industrial robots. In: IEEE International Conference on Automation Science and Engineering, vol. 2014-Janua, pp. 339-344 (2014)

34. Lemaignan, S., Warnier, M., Sisbot, E.A., Clodic, A., Alami, R.: Artificial cognition for social humanrobot interaction: An implementation. Artificial Intelligence 247, 45-69 (2017) 
35. Levesque, H., Lakemeyer, G.: Cognitive Robotics. Foundations of Artificial Intelligence 3(07), 869-886 (2008)

36. Li, K., Fu, Y.: Prediction of human activity by discovering temporal sequence patterns. IEEE Transactions on Pattern Analysis and Machine Intelligence 36(8), 16441657 (2014)

37. Mainprice, J., Sisbot, E.A., Jaillet, L., Cortés, J., Alami, R., Siméon, T.: Planning human-aware motions using a sampling-based costmap planner. In: Proceedings - IEEE International Conference on Robotics and Automation, pp. 5012-5017 (2011)

38. Martín, F., Agüero, C., Cañas, J.M., Abella, G., Benítez, R., Rivero, S., Valenti, M., Martínez-Martín, P.: Robots in Therapy for Dementia Patients. Journal of Physical Agents 7(1) (2013)

39. Matthias, B., Reisinger, T.: Example Application of ISO/TS 15066 to a Collaborative Assembly Scenario. In: In Proceedings of the 47th International Symposium on Robotics, pp. 1-5 (2016)

40. McCallum, S., Boletsis, C.: Dementia games: A literature review of dementia-related serious games. In: Lecture Notes in Computer Science (including subseries Lecture Notes in Artificial Intelligence and Lecture Notes in Bioinformatics) 2013, vol. 8101 LNCS, pp. 15-27 (2013)

41. McColl, D., Nejat, G.: Meal-Time with a Socially Assistive Robot and Older Adults at a Long-term Care Facility. Journal of Human-Robot Interaction 2(1), 152-171 (2013)

42. Mohan, V., Morasso, P., Sandini, G., Kasderidis, S.: Inference Through Embodied Simulation in Cognitive Robots. Cognitive Computation 5(3), 355-382 (2013)

43. Nikolaidis, S., Lasota, P., Rossano, G., Martinez, C., Fuhlbrigge, T., Shah, J.: Human-robot collaboration in manufacturing: Quantitative evaluation of predictable, convergent joint action. In: 2013 44th International Symposium on Robotics, ISR 2013, pp. 1-6. IEEE (2013)

44. Pérula-Martínez, R., Castro-González, ., Malfaz, M., Alonso-Martín, F., Salichs, M.A.: Bioinspired decisionmaking for a socially interactive robot. Cognitive Systems Research 54, 287-301 (2019)

45. Ragaglia, M., Bascetta, L., Rocco, P., Zanchettin, A.M.: Integration of perception, control and injury knowledge for safe human-robot interaction. In: 2014 IEEE International Conference on Robotics and Automation (ICRA), pp. 1196-1202. IEEE (2014)

46. Salichs, E., Fernández-Rodicio, E., Castillo, J.C., CastroGonzález, ., Malfaz, M., Salichs, M..: A Social Robot Assisting in Cognitive Stimulation Therapy. In: Y. Demazeau, B. An, J. Bajo, A. Fernández-Caballero (eds.) Advances in Practical Applications of Agents, MultiAgent Systems, and Complexity: The PAAMS Collection, pp. 344-347. Springer International Publishing (2018)

47. Sheikh, Z.C., Simon, T., Wei, S.E., Yaser: Realtime Multi-Person 2D Pose Estimation using Part Affinity Fields. CVPR (2017)

48. Sisbot, E.A., Alami, R.: A human-aware manipulation planner. IEEE Transactions on Robotics 28(5), 10451057 (2012)
49. Sisbot, E.A., Marin-Urias, L.F., Broquère, X., Sidobre, D., Alami, R.: Synthesizing robot motions adapted to human presence: A planning and control framework for safe and socially acceptable robot motions. International Journal of Social Robotics 2(3), 329-343 (2010)

50. Tapus, A., Mataric, M.: Socially assistive robotic music therapist for maintaining attention of older adults with cognitive impairments. Proceedings of AAAI Fall Symposium AI in Eldercare (January 2008), 124-127 (2008)

51. Tapus, A., Mataric, M., Scassellati, B.: Socially assistive robotics [Grand challenges of robotics]. IEEE Robotics and Automation Magazine 14(1), 35-42 (2007) 